\title{
Spectral analysis of two Perseid meteors
}

\author{
J. Borovička ${ }^{1}$ and H. Betlem ${ }^{2}$ \\ ${ }^{1}$ Astronomical Institute, Ondřejov Observatory, 25165 Ondřejov, Czech Republic \\ ${ }^{2}$ Dutch Meteor Society, Lederkarper 4, NL-2312 NB Leiden, The Netherlands
}

Received for publication 28 January 1997

\begin{abstract}
Spectra of two bright (-1/ mag) Perseid meteers are studied. Monochromatie light curves are constructed and the spectra are analyzed at selected points along the trajectom. The shift of haxima of low excitation iron lines down the trajectory in meteor flares is observed and explained by a longer radiative lifetime of the upper levels for these ines. Two spectral compoments with the temperatures of $4400-4800 \mathrm{~K}$ and $10,000 \mathrm{~K}$, respectively are tentified in the spectra in accordance with previous findings The ratio of both components, in terns of mass, varied smoothly from about $100 / 1$ over 15,1 to 30,1 . This ratio is not an unambiguous function of meteor velocity, height and brightness but depends on the previous evolution of ablation. The abundances of leavy elements are found consistent with the chemical composition of carbonaceous chondrites and the dust of comet Halley Mydrogen, however, is not more abundant than in car bonaceous chondrites and thus significantly less than in cometary dust the witial masses of the two meteoroids are estinated at 40 and $80 \mathrm{~g}$, respectively. The meteor $V$ band luminous effeicney is found to vary in the range $\log \tau=-118$ to -12.2 in magnitide c.gs. units. For the panchromatic luminous efficiency use of the value of 11,4 for bright Persedds s recommended Nearly $15 \%$ of meteoroid kinetie energy is radiated out in the Ca II lines and $1 \%$ in all other lines between 3500 and $6600 \mathrm{~A}$, 1997 Jisevier Seience LtI
\end{abstract}

\section{Introduction}

The Perseid meteor shower is a major shower for northern hemisphere observers with a reliable display peaking around August 12 each year. This makes the Perseids one of the most common targets of meteor observations (including spectroscopy). The meteoroids are derived

Correspondence to: J. Borovička from comet $109 \mathrm{P} /$ Swift-Tuttle and enter the Earth's atmosphere with a large velocity of $60 \mathrm{~km} \mathrm{~s}^{-1}$. Very bright meteors are observed from time to time and if recorded with an objective grating photographic camera, their spectra can be studied in considerable detail.

Halliday (1961) published the most complete list of spectral lines identified in Perseid spectra. Using various photographic emulsions and cameras with dispersion from 50 to $120 \AA \mathrm{Am}^{-1}$, he was able to identify 229 emission features in the region from 3680 to $8710 \AA$ based on a combination of five meteor spectra. Later Halliday (1969) gave the line identifications down to $3100 \AA$ based on one Lyrid and one Perseid spectrum. The ultraviolet lines in a Perseid spectrum were listed also by Harvey (1973a). Cook et al. (1971) performed photometric calibration of two Perseid spectrograms and were able to give line intensities in absolute units. In addition to these photographic works, which dealt with very bright meteors of magnitudes around -10 , spectra of fainter Perseids (magnitudes around 0) were observed with lower dispersion using television techniques (Millman et al., 1971; Borovička and Boček, 1995).

All photographic observations gave the same general picture of a bright Perseid spectrum: the $\mathrm{H}$ and $\mathrm{K}$ lines of ionized calcium at 3934 and $3968 \AA$ are the brightest lines. Other bright lines include ionized magnesium at $4481 \AA$, neutral magnesium triplet at $5167-5184 \AA$, neutral sodium doublet at $5890-5896 \AA$ and neutral oxygen triplet at $77727775 \AA$. Numerous lines of ncutral iron are also present. Other atoms and ions reliably identified are $\mathrm{HI}$, N I, N II, AlI, Si II, Ca I, Cr I, Mn I, Fe II, Ni I, and Sr II. The identification of OII, SiI, SrI, BaI, and BaII in Perseid spectra remained doubtful. On the other hand, the presence of the first positive bands of the nitrogen molecule has been well established.

An indication that the $\mathrm{Ca} I I$ lines are fainter relative to $\mathrm{OI}$ in fainter meteors was noted (Millman and Halliday, 1961). In faint TV spectra the Ca II lines are no more dominant (if present at all) and the brightest are the lines of $\mathrm{OI}$ and $\mathrm{MgI}$. In bright meteors it was noted that the brightness of the Ca II lines relative to O I increased along 
the trajectory and that $\mathrm{Ca}$ II is especially strong in terminal flares (Millman and Halliday, 1961; Halliday, 1961). Cook et al. (1971) mentioned that also the H I and Si II lines increase in strength relative to the rest of the spectrum down the meteoroid trajectory. Borovička and Boček (1995) observed a Perseid flare with a TV camera. All meteoric emissions increased in the flare much more dramatically than the emissions of atmospheric origin due to O I and N I. Another effect in a Perseid flare was observed by Russel (1960): The low excitation lines of neutral iron had their maxima shifted to lower heights with respect to other lines.

In general Perseid spectra are similar to the spectra of other fast meteors. For example the Orionids $\left(67 \mathrm{~km} \mathrm{~s}^{-1}\right)$ show the same lines and spectral development along the trajectory as the Perseids (Halliday, 1987). Since there are more good quality spectra available for Perseids than for any other shower, Perseid spectra can be used to study the formation of the fast meteor spectra and can serve as clues for their interprctation. Possible minor differences in spectra could then reveal possible chemical or structural differences among meteoroids of different showers or within one shower.

Quantitative analyses of Perseid spectra are, however, much rarer in the literature than qualitative descriptions. Harvey (1973a) assumed Boltzmann distribution of level populations and optically thin radiation in his analysis. He computed the effective radiation temperature from the intensity ratios in pairs of lines. The temperature near $3000 \mathrm{~K}$ was derived for Fe I lines in one Perseid spectrum but quite different temperatures were found for three other atoms. The elemental abundances corrected for partial ionization and dissociation were later given in Harvey (1973b). However, the assumption of optical thinness is inadequate for meteors as bright as magnitude -9 (Borovička, 1993) and the simple method used by Harvey cannot be considered as yielding reliable results.

Only few lines were measurable in the Perseid spectra analyzed by Kokchirova (1993). She modified the method of Harvey (1973a) by using the intensity ratios of lines of different elements and assuming the chemical composition. However, the ionization of elements like sodium was not taken into account and the obtained excitation temperatures in the wide range of $1500-12,000 \mathrm{~K}$ have, in our opinion, no physical meaning.

A new method of meteor spectra analysis was developed by Borovička (1993) in connection with the study of a slow fireball. A synthetic spectrum was computed and the free parameters - excitation temperature, column densities of various atoms, and effective cross-section of the radiating volume - were adjusted by the least squares method. Following Ceplecha's method (Ceplecha, 1964) of emission curve of growth, self-absorption was fully taken into account and the Boltzmann population of levels was assumed. It was found that the spectrum can be well explained by assuming two components of quite different temperature.

The two spectral components were studied in more detail by Borovička (1994) in four bright meteors of different velocities. The two spectral components were present in all four meteors with nearly the same temperatures, $4000-4500 \mathrm{~K}$ for the so-called main spectrum and about $10,000 \mathrm{~K}$ for the so-called second spectrum.
Table 1. Atmospheric trajectory of two Perseid fireballs

\begin{tabular}{lcc}
\hline Meteor No. & DMS-85016 & DMS-89046 \\
Date & 1985 Aug. 12 & 1989 Aug. 13 \\
Time UT & $2 \mathrm{~h} 11$ min 20s & 2h 27 min 40 s \\
\hline$h_{\mathrm{B}}(\mathrm{km})$ & 117.5 & 108.1 \\
$h_{\mathrm{E}}(\mathrm{km})$ & 70.3 & 72.6 \\
$v_{\infty}\left(\mathrm{km} \mathrm{s}^{-1}\right)$ & $60.6 \pm 0.4$ & $60.9 \pm 0.5$ \\
$v_{\mathrm{E}}\left(\mathrm{km} \mathrm{s}^{-1}\right)$ & $57 \pm 2$ & $58 \pm 2$ \\
$z_{\mathrm{R}}$ & $28^{\circ} .8$ & $27^{\circ} .0$ \\
$L(\mathrm{~km})$ & 53.6 & 39.8 \\
$D(\mathrm{~s})$ & 0.90 & 0.66 \\
\hline
\end{tabular}

Explanation of symbols: $h_{\mathrm{B}}$, beginning height; $h_{\mathrm{E}}$, terminal height; $v_{\infty}$, initial velocity; $v_{\mathrm{E}}$, terminal velocity; $z_{\mathrm{R}}$, zenith distance of the radiant; $L$, total trajectory length; $D$, total duration.

The main spectrum contains lines of neutral atoms, especially $\mathrm{Na}, \mathrm{Mg}, \mathrm{Fe}, \mathrm{Ca}, \mathrm{Cr}, \mathrm{Mn}, \mathrm{Al}$ and ionized $\mathrm{Ca}$. In the second spectrum most metals are ionized and the main contributing lines are those of ionized $\mathrm{Ca}, \mathrm{Mg}, \mathrm{Si}, \mathrm{Fe}$ and neutral $\mathrm{O}, \mathrm{N}, \mathrm{H}$ (which have high ionization potential). The main result was that the ratio of the mass of the gas involved in the production of the main and the second spectrum, respectively, $m_{1} / m_{2}$, is a strong function of velocity. It was about 3000 in the slow $17 \mathrm{~km} \mathrm{~s}^{-1}$ fireball but decreased to 15 in a $67 \mathrm{~km} \mathrm{~s}^{-1}$ Orionid. Different strength of the second component is the main factor which discriminate the spectra of slow and fast fireballs. It is probable that the second component is related to the shock wave formed in front of the meteoroid.

The analysis in Borovička (1994) was restricted to the brightest points of four fireballs. The aim of this paper is to study the development of both spectral components along the trajectory. For this purpose two spectra of bright Perseids photographed in the Netherlands were analyzed. This is the first quantitative analysis of Perseid spectra which recognizes the two components and takes the self-absorption fully into account. The analyzed spectra are also used to derive chemical composition of Perseid meteoroids and to study the luminous efficiency of fast meteors.

\section{Observational material}

The meteors analyzed here are DMS-85016 (Dutch Meteor Society meteor No. 16 in 1985) of August 12, 1985 and DMS-89046 of August 13, 1989. Both were photographed by a network of directly imaging cameras equipped with rotating shutters which enabled the trajectory, velocity and orbit to be fully determined. The meteors have been described in more detail in Betlem and de Lignie (1985) and van Oudheusden and van Dijk (1991), respectively. A summary of the data on their atmospheric trajectories is given in Table 1. An absolute photographic magnitude of about -11 was reached in both cases. The photometry and associated problems are, however, discussed below in connection with the luminous efficiency.

The spectra were obtained at station Bussloo, in the Netherlands with a $f=16 \mathrm{~cm}$ Zeiss Tessat camera and a 400 grooves $\mathrm{mm}^{-1}$ grating using Kodak Tri-X sheet film. No shutter was present. The spectrum of DMS-85016 (here- 




Fig. 1. The spectrum of meteor DMS-85016 (spectrum A). The meteor flew from top left to bottom right. Part of the zero order image is visible on the right. The bright lines near the blue end of the spectrum are visible also in the second order 




Fig. 2. Relative sensitivity of our spectrograph as a function of wavelength. Normalized at $6500 \AA$

after referred to as spectrum A) is reproduced in Fig. 1. The transmissivity of the optics and the sensitivity of the photographic material enabled the wavelengths from 3500 to $6600 \AA$ to be covered. The spectral sensitivity of the system was determined by measuring the spectrum of $\alpha$ Lyrae recorded in the same frame as spectrum $A$ and dividing it by the known energy distribution in the A0 V star spectrum (Sviderskiene, 1988). The sensitivity function for the first spectral order is given in Fig. 2. As the distances of the meteors from the camera (nearly $185 \mathrm{~km}$ for the bright parts of both meteors) and the meteor angular velocities $\left(18^{\circ} \mathrm{s}^{-1}\right)$ were known, the absolute calibration of the spectra was possible. Owing to the angle of only about $30^{\circ}$ between the meteor direction and the dispersion line, the effective dispersion was approximately $300 \hat{\mathrm{A} \mathrm{mm}} \mathrm{m}^{-1}$ in both cases.

The light curves of both meteors are similar. For a long time the meteors are relatively faint and the brightness increases slowly. Then the brightness rises abruptly and a period of intensive radiation follows. Toward the end the meteors become fainter with several distinctive flares of moderate brightness. A sufficient number of lines in the spectra is available only during the period of intensive radiation and the detailed analysis will therefore be restricted to this part of the trajectory.

\section{Line identifications and monochromatic light curves}

Both spectra are remarkably similar and are quite typical for Perseid meteors. The $\mathrm{H}$ and $\mathrm{K}$ lines are by far the most intense features along the whole trajectory. At the beginning, they are the only visible lines. The infrared oxygen lines, which could be of comparable brightness at the beginning, lie outside our spectral range. The other bright lines present in the visual region are also well known from the spectra of Perseids and other fast meteors.

The identification of the main spectral features is given here in Fig. 3 where the two spectral components, computed by the method of Borovička (1993), are also shown. The tracing of spectrum $A$ in the flare at the height of $79.5 \mathrm{~km}$ is given in the upper part of the figure. The computed synthetic spectra of the main component (temperature $T=4800 \mathrm{~K}$, column density of neutral iron $N_{\mathrm{FeI}}=1.5 \times 10^{15} \mathrm{~cm}^{-2}$ ) and the second component
$\left(T=10,000 \mathrm{~K}, N_{\text {Fe II }}=10^{14} \mathrm{~cm}^{-2}\right)$ are given below it. The lines are identified by the name of atom/ion. For a more detailed identification see the list of observed lines in Halliday (1961) or of computed lines in Borovička (1994). The Ca II lines are produced mostly in the second spectrum although they are predicted to be present also in the main component.

In addition to the species mentioned in Fig. 3, also Cr II (multiplet 44) and TiII (82) may contribute to the lines observed at 4550 and $4585 \AA$, together with Fe II (38) which is the main contributor. The $H \beta$ line at $4861 \hat{A}$ may be present as a faint feature near the Fe I line. Cr II, Ti II and $\mathrm{HI}$ all belong to the second spectrum.

Two methods were used to study the temporal evolution of the spectral components. Firstly, several lines were measured along the trajectory and monochromatic light curves as a function of height were obtained. Secondly, the spectrum was completely analyzed at selected points along the trajectory and the mass ratio of both components of gas was determined. Both approaches were restricted to the bright parts of the meteor trajectory.

Representative monochromatic light curves are given in Fig. 4. The main spectrum is represented by the sodium doublet. Other lines of the main component behaved similarly except for the low multiplets of iron (see below). In spectrum $A$ a broad maximum occurred between 85 and $82 \mathrm{~km}$ and two shorter flares are present at 79.5 and $78.5 \mathrm{~km}$. In spectrum B the former maximum is missing completely, two bright flares are present at 79 and $78 \mathrm{~km}$ and one fainter at $77 \mathrm{~km}$.

The lines of iron multiplets 1 and 2 show the effect described by Russel (1960); the flares are shifted down the trajectory relative to the other lines. This is clearly visible by a visual inspection of original negatives and also in Fig. 4 where the light curve of one Fe I (2) line is presented. The multiplets 1 and 2 are low excitation intercombination lines originating from metastable levels and are, as noted already by Russel (1960), typical for the radiation of the meteor wake. Other intercombination lines, e.g. Mg I multiplet 1, would probably show the same effect, if they were clearly visible in the spectrum.

The explanation of the strength of intercombination lines in the wake and the shift of their flares lies probably in slower de-excitation of metastable levels after the atoms lcave the hot radiating volume. We present here a possible qualitative explanation of the Russel effect. The situation in a flare is demonstrated schematically in Fig. 5. The physical processes involved are shown in the upper part of the figure. The time history of an individual atom can be read by drawing a horizontal line. The flare starts as a result of subsequent evaporation of a large amount of material in a short time interval. After evaporation, the atoms reach a hot region of gas in nearly thermal equilibrium (TE) with Boltzmann population of levels maintained by mutual collisions of atoms, ions and free electrons. This region is the main source of the main spectrum radiation. After a time (sometimes called the relaxation time) atoms leave the hot region and reach a region where the collisional frequency decreases. There is no mechanism to maintain stable populations of excited levels and atoms de-excite, primarily by radiative transitions. The low-lying metastable levels, however, de-excite more slowly because of smaller transition probability from them. Nevertheless, 

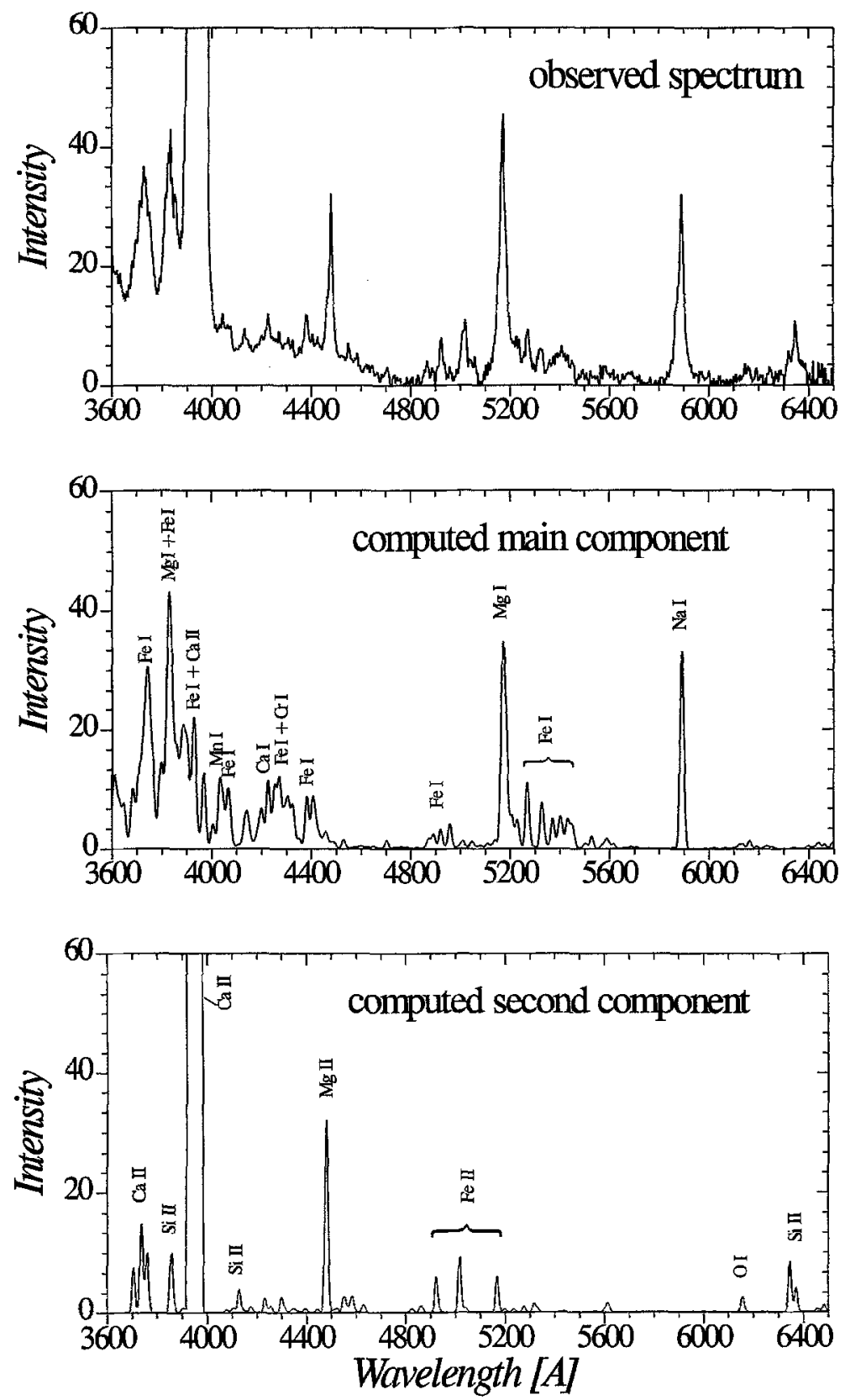

Fig. 3. The calibrated photometric tracing of spectrum $A$ at the height of $79.7 \mathrm{~km}$ (above) and the computed synthetic spectra of the main and second spectral components. The observed spectrum is slightly out of focus above $5500 \AA$. The intensity scale is in units of $10^{9} \mathrm{erg} \mathrm{s}^{-1} \AA^{-1} \mathrm{sr}^{-1}$ while the instrumental width of individual lines is about $10 \AA$. The Ca II lines are out of the given scale, their computed intensities in the second spectrum are 750 and 500 . The observed intensities are about these values or more, the calibration is uncertain due to overexposure

the effective lifetime must not be exactly the radiative lifetime of the levels because some collisional excitation from the ground state can still occur. Moreover, the level population is also enhanced by downward transitions from higher levels.

In the lower part of Fig. 5, the number of allowed and intercombination radiative transitions in the whole meteor is shown as a function of time. For an optically thin line this number is directly proportional to the line intensity. For an optically thick line the situation is more complicated and depends on geometry but the overall picture will be the same. Before the flare, the allowed and intercombination lines are at their (different) quiet intensities. In the flare, the number of allowed transitions is proportional to the number of atoms in the TE region. Similarly, the number of intercombination transitions is proportional to the population of metastable levels. At the final de-excitation of the highly excited levels, the population of the metastable levels is enhanced above its equilibrium value by downward cascade transitions from the high levels. These downward transitions are, of course, present also before but they are not important in the TE region because the level populations are maintained here by much more frequent collisional transitions. 

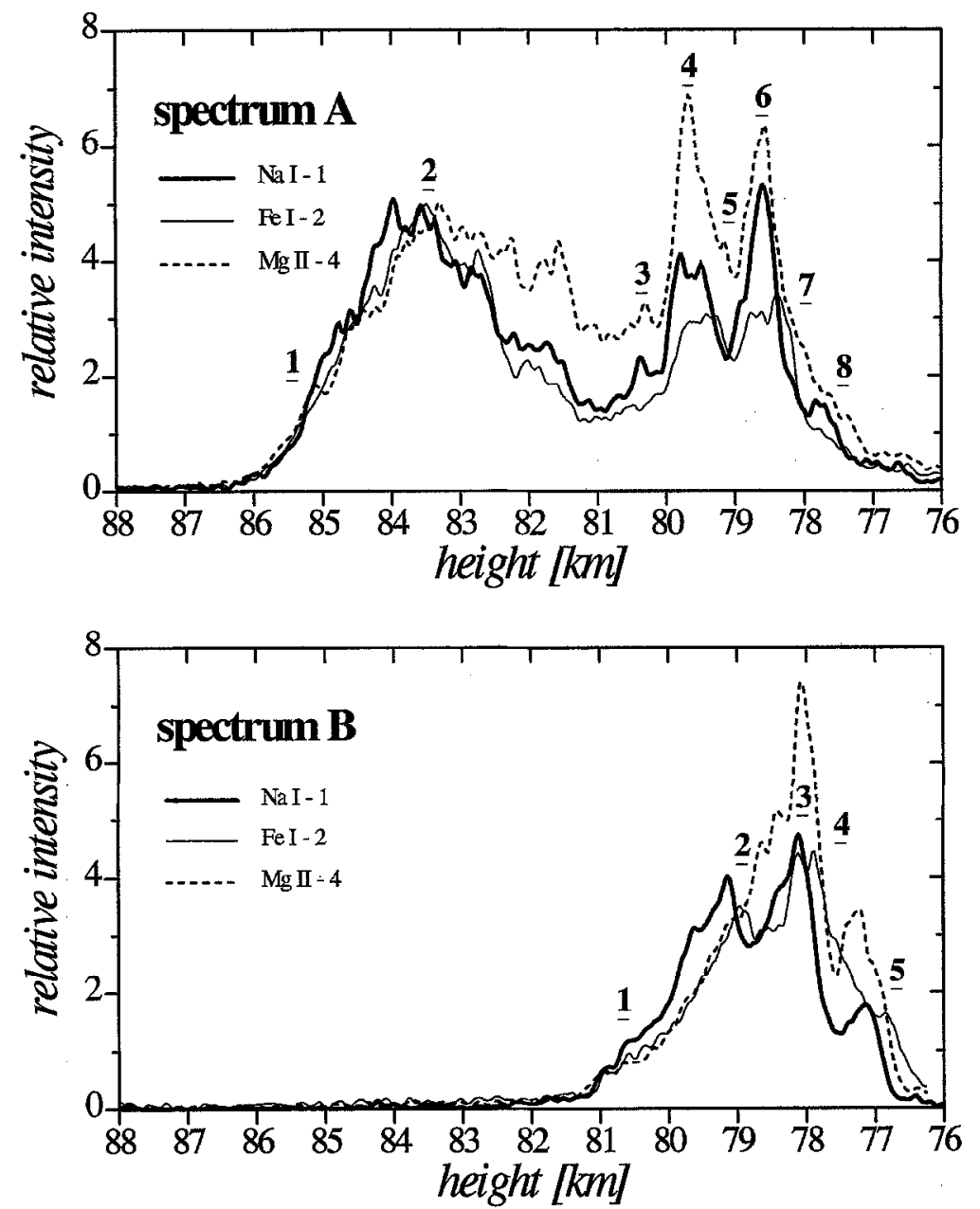

Fig. 4. Monochromatic light curves in three representative spectral lines: the unresolved sodium doublet ( 5890 and $5896 \AA)$, the Fe I multiplet 2 line $(4427 \AA)$, and the Mg II line $(4481 \AA)$. The curves have been normalized to reach the same intensity around $84 \mathrm{~km}$ of height in spectrum $A$ and around $79 \mathrm{~km}$ in spectrum B. The underlined numbers designate the points where the spectrum has been analyzed (see Table 2)

The final relation of the two lines in Fig. 5 is similar to what is observed, especially in the flare at $79.5 \mathrm{~km}$ in spectrum A (Fig. 4). Things are certainly more complex in reality than shown here, but it has been demonstrated at least that the temporal shift of the intercombination lines is understandable. There is no reason to consider the intercombination lines as a distinctive spectral component, they are excited in the same region as the other lines of the main component.

The second component is represented in Fig. 4 by the line of the ionized magnesium. It is clearly seen that the $\mathrm{Mg}$ II line, in comparison with $\mathrm{Na} \mathrm{I}$, is relatively faint at the beginning and bright toward the end. Of particular interest is the comparison of spectra $\mathrm{A}$ and $\mathrm{B}$ below the height of $80 \mathrm{~km}$, where two flares of $\mathrm{Na} I$ are present in both cases. While the $\mathrm{Mg}$ II line is faint during the first flare in spectrum $B$, in spectrum $A$ (where one bright maximum already occurred at about $84 \mathrm{~km}$ ) $\mathrm{MgII}$ is bright in both flares. This demonstrates that the intensity ratio of both lines is not an unambiguous function of meteor height, brightness etc. but depends on the history of the meteor. The lines of Si II and Fe II show the same behavior as $\mathrm{Mg}$ II. For $\mathrm{Ca}$ II this behavior seems to be less pronounced but the calibration of the very bright lines represents some problem. The relation of the two spectral components was further studied by the analysis of the spectra at selected points.

\section{Physical analysis of the spectra}

The spectra were analyzed by the method of Borovička (1993) at the points marked in Fig. 4. The resolution of the spectra did not allow quite unambiguous solutions. In particular, the column density and cross-section could not be clearly separated. However, their product, which gives the total number of atoms in the radiating gas, could be obtained with confidence. The effective excitation temperature of the main component gas was determined with the estimated precision of $\pm 200 \mathrm{~K}$. For the second component the temperature of $10,000 \mathrm{~K}$ was found quite satisfactory and was used as fixed for all points. The resulting temperature, $T$, of the main component, total number of neutral iron atoms, $N_{\mathrm{FeI}}$, in the main component, and iron ions, $N_{\mathrm{FeI}}$, in the second component gas are given in Table 2.

The temperature of the main component was found 


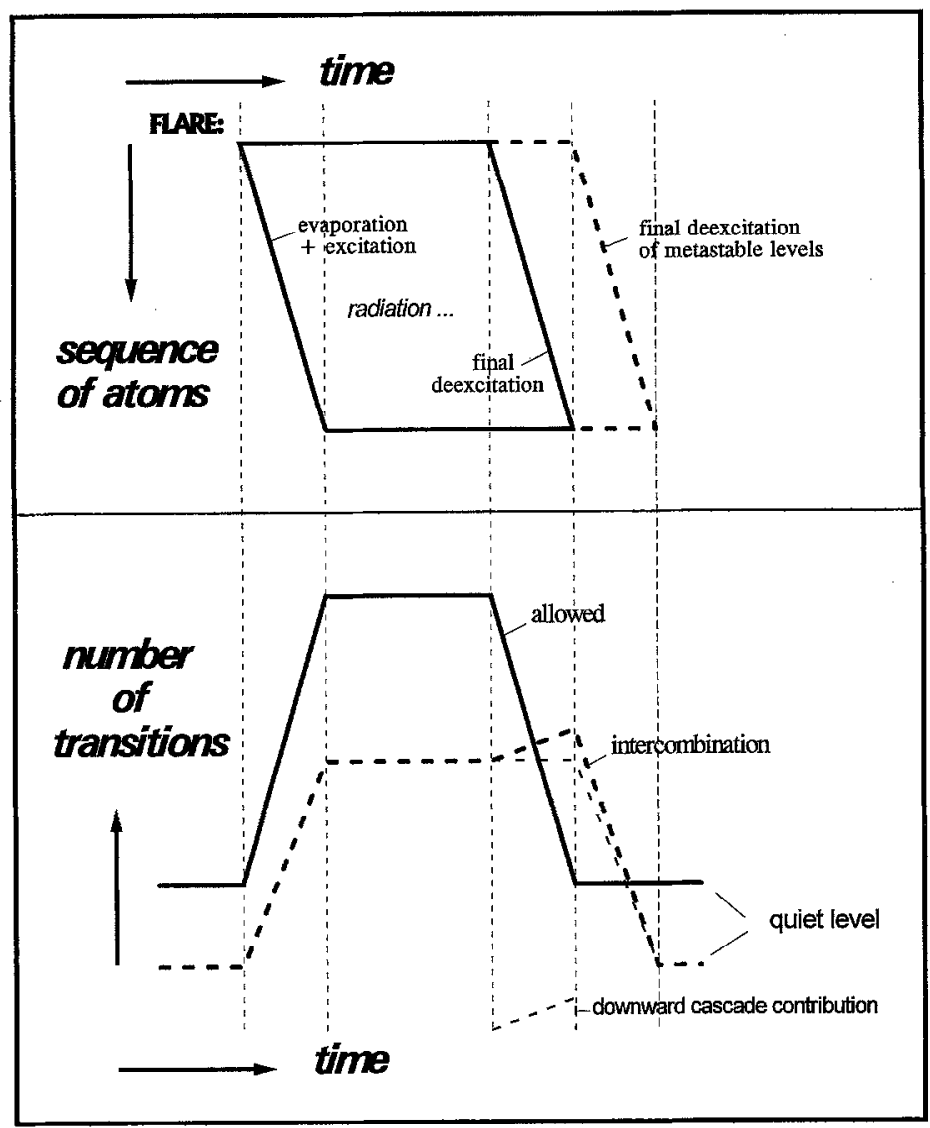

Fig. 5. Schematic representation of a meteor flare and the explanation of the shift of maxima of intercombination lines. See the text for more explanation

Table 2. Two spectral components in Perseid spectra

\begin{tabular}{|c|c|c|c|c|c|c|}
\hline \multirow[t]{2}{*}{ Point No. } & \multirow[t]{2}{*}{ Height $(\mathrm{km})$} & \multicolumn{3}{|c|}{ Main component } & \multirow{2}{*}{$\begin{array}{c}\begin{array}{c}\text { Second } \\
\text { component }\end{array} \\
N_{\mathrm{FerI}}=N_{\mathrm{Fe}} \\
\left(\times 10^{19}\right)\end{array}$} & \multirow{2}{*}{$\begin{array}{l}\text { Ratio } \\
m_{1} / m_{2}\end{array}$} \\
\hline & & $T(\mathrm{~K})$ & $\begin{array}{c}N_{\text {FeI }} \\
\left(\times 10^{19}\right)\end{array}$ & $\begin{array}{c}N_{\mathrm{Fe}} \\
\left(\times 10^{19}\right)\end{array}$ & & \\
\hline \multicolumn{7}{|c|}{ Spectrum $A$} \\
\hline 1 & 85.2 & 4650 & 530 & 1300 & 18 & 70 \\
\hline 2 & 83.6 & 4600 & 1300 & 2500 & 35 & 70 \\
\hline 3 & 80.3 & 4800 & 200 & 500 & 30 & 16 \\
\hline 4 & 79.7 & 4800 & 390 & 800 & 60 & 13 \\
\hline 5 & 79.1 & 4650 & 400 & 800 & 40 & 20 \\
\hline 6 & 78.6 & 4700 & 850 & 1700 & 60 & 28 \\
\hline 7 & 78.0 & 4750 & 350 & 800 & 30 & 28 \\
\hline 8 & 77.5 & 4450 & 380 & 600 & 17 & 35 \\
\hline \multicolumn{7}{|c|}{ Spectrum B } \\
\hline 1 & 80.5 & 4350 & 540 & 900 & 9 & 100 \\
\hline 2 & 79.0 & 4550 & 600 & 1200 & 30 & 40 \\
\hline 3 & 78.1 & 4750 & 450 & 1100 & 55 & 20 \\
\hline 4 & 77.4 & 4550 & 260 & 550 & 20 & 27 \\
\hline 5 & 76.7 & 4850 & 140 & 500 & 15 & 33 \\
\hline \multicolumn{7}{|c|}{ Meteor No. 221 (Cook et al., 1971) } \\
\hline 6.2 & 87.9 & 4600 & 750 & 2100 & 100 & 21 \\
\hline 7.2 & 83.8 & 4600 & 600 & 1700 & 70 & 24 \\
\hline \multicolumn{7}{|c|}{ Meteor No. 233 (Cook et al., 1971) } \\
\hline 6.1 & 81.8 & 4600 & 500 & 1500 & 60 & 25 \\
\hline
\end{tabular}


typically between 4500 and $4800 \mathrm{~K}$. This is in the upper part of the range derived in previous meteor analyses with the same method (Borovička, 1993, 1994; Borovička and Spurný, 1996). The fact that temperatures of both spectral components do not depend on meteor velocity was confirmed again.

To obtain the ratio of the mass of ablation products involved in the production of both components, we can use the ratio of the number of iron atoms as the mass percentage of iron is assumed to be the same in both components and equal to the iron mass percentage in the meteoroid. However, only neutral iron is observed in the main spectrum and only ionized iron is observed in the second spectrum. We have therefore to estimate the ionization degree of iron to obtain the total iron content. The ionization degree depends on temperature and the density of free electrons. The temperature is known, the electron density can be computed by the method described in Borovička (1993) under some assumptions as to the geometry of the radiating volume and provided that the abundances of the elements contributing most of the free electrons are known.

For the second spectrum it was found that almost all iron is singly ionized for a relatively wide range of parameters around the most probable iron density of $10^{14} \mathrm{~cm}^{-3}$ (Borovička, 1994). The derived amount of Fe II is therefore equal to the total amount of iron. The situation is more complicated for the main spectrum. The most probable combinations of column density and crosssection were used to estimate the size and density of the radiating volume. The main contributors of free electrons proved to be magnesium, iron, and to some extent silicon. Silicon is not observable directly in the main spectrum but its contribution could be estimated from the supposed chemical composition. The following range of parameters was then found at various points in the two meteors: diameter of the radiating column, $8-16 \mathrm{~m}$, and electron density from $3 \times 10^{12}$ to $2 \times 10^{13} \mathrm{~cm}^{-3}$. It followed that 40 $60 \%$ of iron was ionized. The total amount of iron in the main component gas at various points is also given in Table 2. Finally, the ratio of iron content in the main component to the second component is given in the last column.

The ratio is changing in the way already seen in monochromatic light curves. The share of the second component is low at the beginning of the bright part of both meteors, with $m_{1} / m_{2} \approx 70-100$. Then the relative importance of the second component grows and the ratio decreases to 15-20. Toward the end the ratio exceeds 30 again. It should be noted that the high share of the second component is not correlated with meteor flares. This can be seen well in spectrum A. The second component is strong not only in the flare at $79.5 \mathrm{~km}$ but also before this flare. On the contrary, it is two times weaker in relative scale in the subsequent flare at $78.5 \mathrm{~km}$. The ratio of both components changes more slowly than the amount of mass in both components themselves.

To be able to compare the present results with other meteors, two meteors analyzed by Cook et al. (1971) have been evaluated by our procedure, using the absolute intensities of important lines given by the authors. Because of a more limited amount of data available, the reduction was more uncertain. Nevertheless, some estimates have been obtained and are given in Table 2. The items dependent on the ionization correction are most uncertain and are printed in italics. The column density seemed to be lower in these meteors, yielding larger radiating. volume, lower density and higher ionization. This may be a consequence of larger height where these meteors reached their maximum brightness. Nevertheless, the final ratios $m_{1} / m_{2}$ are very similar to those in our meteors.

The mass ratio of both components as a function of meteor velocity was studied by Borovička (1994). Each meteor was, however, analyzed only at its brightest point. The lowest ratio was found in a $67 \mathrm{~km} \mathrm{~s}^{-1}$ Orionid: $m_{1} / m_{2}=15$. The Perseids analyzed here reach comparable values in the middle of their bright part. The ratio, however, changes along the trajectory and the share of the second component is much lower at the beginning. This is the reason for the increase of the brightness of some lines relative to other lines as mentioned by several authors (Cook et al., 1971; Millman and Halliday, 1961).

The observations of other authors show that the lines of the second component are fainter in faint Perseids which radiate at higher height (e.g. Millman et al., 1971). Nevertheless, as mentioned already in the previous section, no function relating directly the ratio of both components with meteor brightness or height (for a given velocity) can be given. In fact, the share of the second spectrum rises with some delay after the ablation rate has increased dramatically. This can be tentatively interpreted as a consequence of development of a strong ablation shock wave. Some modelling would, however, be needed to explain the origin of the second spectral component definitely. Note that Borovička and Boček (1995) observed the line of $\mathrm{Na}$ I to brighten after $\mathrm{Ca}$ II and $\mathrm{Mg} \mathrm{II}$, which is in contradiction with the present observations. However, this could be caused by a low time resolution of $0.04 \mathrm{~s}$. A similar effect would appear for example, if we compared the points 4 and 6 of spectrum A (Fig. 4). They are $0.02 \mathrm{~s}$ apart.

Another interesting question is the behavior of oxygen and nitrogen lines. We observe only one faint oxygen line in our spectra, which is insufficient for detailed analysis. However, the infrared OI and NI lines are common in Perseid spectra. They appear early, do not brighten along the trajectory in such a way as Ca II lines and other lines of the second component, show much smaller amplitude in flares, and are strong also in faint Perseids (Borovička and Boček, 1995; Halliday, 1961 ; Millman and Halliday, 1961). These lines are certainly of atmospheric and not meteoritic origin. They need high temperature for excitation and are in fact fully consistent with being excited in the same region as the second spectrum under the temperature of $10,000 \mathrm{~K}$. The same is valid also for the $\mathrm{N}_{2}$ molecule. We can therefore interpret the situation so that the $10,000 \mathrm{~K}$ region is always present in Perseids and other fast meteors and the relative amount of meteoritic to atmospheric material in it is variable. A large amount of meteoritic material is present only during the high ablation phase in bright meteors. But if we identify the second spectrum region with the shock wave, the question arises, whether the shock wave can be present also at large heights and in faint meteors. These questions are to be studied in the future. It is highly probable that atmospheric material is present in large amounts also in the 
main spectrum region but does not radiate at lower temperatures. Note also that the N II lines observed by Halliday (1961) correspond to a temperature of the order of $30,000 \mathrm{~K}$. These lines are probably not excited by thermal processes.

\section{Chemical composition}

Since we were able to estimate the ionization degree in the radiating gas, we can infer the abundances of the observable elements. In addition to $\mathrm{Fe}$, the column densitics of $\mathrm{Na} \mathrm{I}, \mathrm{MgI}$, Ca I, Cr I and $\mathrm{MnI}$ in the main component could be estimated from the spectra. These column densities are, however, based on only one or a few lines, and in all cases except $\mathrm{NaI}$ the lines were blended with iron lines. It is therefore not surprising that abundances determined at various points showed very large scatter reaching a factor of four between individually determined values. We have therefore computed average relative abundances at points 2-7 in spectrum $A$ and at points 15 in spectrum B.

Also the second component could be used to derive ratios of some elements. A temperature of $10,000 \mathrm{~K}$ was assumed. Since all lines, except $\mathrm{Ca}$ II $\mathrm{H}$ and $\mathrm{K}$, are optically thin, the relative abundances are not dependent on the assumed column densities. The $\mathrm{Ca}$ II abundance was not determined because $\mathrm{H}$ and $\mathrm{K}$ lines are too bright for analysis and the lines of multiplet 3 could not be separated from lines of the main component.

The results for both components are given in Table 3 together with the average abundances in carbonaceous chondrites of type $\mathrm{Cl}$, which are the meteorites with chemical composition most close to the original composition of solar system material (Anders and Grevesse, 1989). The abundances in the dust of comet Halley as obtained from in situ mass spectroscopy (Jessberger et al., 1988 ) is also given for comparison because Perseids are cometary meteors. Iron is used as reference element because it has by far the most numerous lines in meteor spectra and the ratios to iron are the most reliable ratios obtainable from meteor spectroscopy.

Most values in Table 3 are within a factor two of the chondritic and cometary values, which is inside of the expected error. In fact, this consistent result is very satisfying because of the above described difficulties and the fact that the ionization correction was extremely large for some elements. It was computed that $99.5-99.8 \%$ of sodium and $98.599 .3 \%$ of calcium was ionized at various points along the meteor trajectories. In summary, it seems that Perseids have quite normal chemical composition, at least for the observable elements. Possible minor deviations are discussed below.

The good agreement of calcium abundance with meteoritic value is remarkable. In many meteors, calcium is depleted in the radiating gas owing to incomplete evaporation (Borovička, 1993; Borovička and Spurný, 1996). A good example is the Benešov bolide, a stony body of the estimated initial diameter of $2 \mathrm{~m}$ and velocity of $21 \mathrm{~km} \mathrm{~s}^{-1}$ (Borovička and Spurný, 1996). The abundance of calcium in the radiating gas was more than two orders of magnitude lower than in chondrites at the height of $78 \mathrm{~km}$, i.e. a height similar to that of the present meteors. The Benešov bolide, however, reached much lower heights and the calcium abundance was found normal at $25 \mathrm{~km}$. Here, the spectrum was quite unusual owing to the prominent role of $\mathrm{Ca} I$ lines. On the contrary, Perseid spectra do not show unusually bright $\mathrm{Ca} I$ lines because most calcium is ionized owing to the low density of the gas. The complctc evaporation, rcachcd in the Benešov bolide only in dense atmospheric layers, was achieved in Perseids high in the atmosphere, obviously because of their high velocity and a more violent ablation. The temperature of the main component gas was by about $1000 \mathrm{~K}$ higher in Perseid meteors than in the Benešov bolide at similar heights.

A high $\mathrm{Si} / \mathrm{Fe}$ ratio was found in comet Halley and the $\mathrm{Mg} / \mathrm{Fe}$ ratio was also relatively high. The Si abundance as determined from the Perseid second spectral component is also higher than in chondrites, although lower than in Halley. For $\mathrm{Mg}$ the situation is unclear. This is the only element with abundances determined independently from both spectral components. The values differ by a factor of two. The abundance in main component may be slightly overestimated owing to the blend of the bright $\mathrm{MgI}$ line with lines of $\mathrm{Fe} I$ and $\mathrm{Fe}$ II. On the other hand, the abundance derived from the second component depends on the assumed temperature. If the temperature were lower than $10,000 \mathrm{~K}$, the abundances of both $\mathrm{Mg}$ and $\mathrm{Si}$ must have been higher. We conclude that a factor of two lies within the observational errors and that the $\mathrm{Mg}$ and $\mathrm{Si}$ abundances are consistent with chondritic values.

Volatile elements are of particular interest. As N and O comc from the Earth's atmosphere, only hydrogen is evidence of the volatile content of Perseid meteoroids. In contrast to the spectra of Cook et al. (1971), our spectra do not show the $\mathrm{H} \alpha$ line at $6563 \AA$. It lies near the sensitivity limit of the film and the spectrum is not particularly well focused in this region but, nevertheless, the line should be visible. From its absence we can obtain an upper limit to the hydrogen abundance (see Table 3). The faint

Table 3. Relative elemental abundances by number in two Perseid spectra, C1 chondrites and comet IIallcy dust

\begin{tabular}{|c|c|c|c|c|c|c|c|c|c|c|c|}
\hline & & $\mathrm{Fe}$ & $\mathrm{Na}$ & $\mathrm{Mg}$ & $\mathrm{Si}$ & $\mathrm{Ca}$ & $\mathrm{Cr}$ & Mn & $\mathrm{H}$ & $\mathbf{N}$ & $\mathrm{O}$ \\
\hline \multirow[t]{2}{*}{ spec. A } & main component & 1 & 0.10 & 2.2 & & 0.06 & 0.010 & 0.014 & & & \\
\hline & 2nd component & 1 & & 1.0 & 2.5 & & & & 2 & $<50$ & 30 \\
\hline & 2nd component & 1 & & 1.1 & 2.0 & & & & 3 & $<50$ & 30 \\
\hline C1 chondrites ${ }^{\mathrm{a}}$ & & 1 & 0.06 & 1.2 & 1.1 & 0.07 & 0.015 & 0.011 & 6 & 0.07 & 9 \\
\hline 1P/Halley dust ${ }^{b}$ & & 1 & 0.19 & 1.9 & 3.5 & 0.12 & 0.017 & 0.010 & 40 & 0.8 & 17 \\
\hline
\end{tabular}

${ }^{a}$ Anders and Grevesse (1989).

${ }^{\mathrm{b}}$ Jessberger et al. (1988) as cited in Anders and Grevesse (1989). 
$\mathrm{H} \beta$ line is nearly consistent with this limiting value, which may therefore represent the real $\mathrm{H}$ abundance. It is lower than in carbonaceous chondrites. Analyzing the spectra of Cook et al. (1971), we found values of $\mathrm{H} / \mathrm{Fe}=4-6$, consistent with chondritic value. Using the $\mathrm{H} \beta$ line in the TVS 29 spectrum of Borovička and Boček (1995), we found $\mathrm{H} / \mathrm{Fe} \approx 5$. It can therefore be concluded that the Perseid spectra analyzed so far show hydrogen abundance comparable or lower than in carbonaceous chondrites and significantly lower than in comet Halley dust. This can be easily explained by considering that meteoroids released from the parent comet lose their volatiles when they are orbiting the Sun.

The upper limit of the nitrogen abundance in the second component gas, derived from the absence of NI lines near $5610 \AA$, is relatively low when compared with oxygen. The ratio $\mathrm{N} / \mathrm{O} \leq 1.5$ is obtained which can be compared with $\mathrm{N} / \mathrm{O} \approx 4$ in the Earth's atmosphere. One possible explanation could be that a substantial part of the oxygen in the spectrum is of meteoritic origin. This would, however, need $\mathrm{O} / \mathrm{Fe}>18$ in the meteoroid which seems improbable considering the low content of hydrogen. Another explanation is that due to the high dissociation potential of the $\mathrm{N}_{2}$ molecule $(9.8 \mathrm{eV})$, a large fraction of nitrogen is in molecular form. Indeed, molecular bands of $\mathrm{N}_{2}$ are commonly seen in Perseid spectra (Cook et al., 1971; Borovička and Boðek, 1995). $\mathrm{O}_{2}$ has much lower dissociation potential $(5.1 \mathrm{eV})$ but the absence of $\mathrm{O}_{2}$ bands is not conclusive because no strong bands of $\mathrm{O}_{2}$ exist in the appropriate spectral region. In any case, it would be better to determine the $\mathrm{N}$ and $\mathrm{O}$ abundances from the strong infrared lines. Published IR spectra have, however, not been calibrated. The estimate for the TVS 29 spectrum (Borovička and Boček, 1995 ) gives $\mathrm{N} / \mathrm{O} \approx 2.5$, showing that the problem may be less severe in this case.

\section{Luminous efficicncy}

The luminous efficiency, $\tau$, expresses which part of the kinetic energy lost by a meteoroid ablating in the atmosphere is being converted into radiation. It is found in the classical luminosity equation :

$$
I=-\tau \frac{v^{2}}{2} \frac{\mathrm{d} m}{\mathrm{~d} t}
$$

where $I$ is the meteor's luminosity, $v$ its velocity, $m$ its mass and $t$ time. By integrating equation (1), the unknown meteoroid initial mass, $m_{\infty}$, can be determined from the known luminosity and velocity as a function of time. For this purpose, luminosity is usually expressed in magnitude based units

$$
I=10^{-0.4 M}
$$

where $M$ is the absolute $(100 \mathrm{~km}$ distance) meteor magnitude. Velocity is given in $\mathrm{cm} \mathrm{s}^{-1}$ and mass in grams. The mass determined this way is called photometric mass. For fast cometary meteors it is the only possibility to determine meteoroid mass because the dynamic mass cannot be derived owing to negligible deceleration.

The luminous efficiency is, however, a poorly known quantity. It depends on velocity. For velocities larger than
$27 \mathrm{~km} \mathrm{~s}^{-1}$, the formula of Ceplecha and McCrosky (1976) is usually used:

$$
\log \tau=-13.69+\log v
$$

where $v$ is given in $\mathrm{km} \mathrm{s}^{-1}$. This gives $\log \tau=-11.9$ for Perseids $\left(v=60 \mathrm{~km} \mathrm{~s}^{-1}\right)$. We will try to determine the luminous efficiency independently from the Perseid spectra analyzed in the present paper.

To estimate the mass loss rate, we use the ratio of the instantaneous mass of the meteoritic part of the radiating gas to the relaxation time (i.e. the time spent by the ablated atoms in the radiating gas):

$$
\frac{\mathrm{d} m}{\mathrm{~d} t} \approx \frac{m_{\mathrm{G}}}{t_{\mathrm{R}}} .
$$

The relaxation time can be estimated from a meteor flare as the interval between the flare onset and the beginning of the decline (Fig. 5). For the flare at $79.5 \mathrm{~km}$ in spectrum A $t_{\mathrm{R}} \approx 0.011 \mathrm{~s}$ was found. The mass of the radiating gas can be determined from the total number of iron atoms, $N_{\mathrm{Fe}}$, as given in Table 2:

$$
m_{\mathrm{G}}=\frac{N_{\mathrm{Fe}} A_{\mathrm{Fe}}}{f_{\mathrm{Fe}}}
$$

where $A_{\mathrm{Fe}}=9.3 \times 10^{-23} \mathrm{~g}$ is the mass of iron atom and $f_{\mathrm{Fe}}$ is the mass fraction of iron in the meteoroid. We assume $f_{\mathrm{Fe}}=18.5 \%$ as in carbonaceous chondrites (Anders and Grevesse, 1989).

Even though some doubts can be raised on the correctness of the estimation of $\mathrm{d} m / \mathrm{d} t$, the most problematic quantity is, paradoxically, the meteor magnitude $M$. Unlike slow and bright meteors, where numerous optically thick lines are present and the spectrum approaches a black body spectrum (Borovička, 1993; Borovička and Spurný, 1996), the luminosity of bright Perseids and other fast meteors is governed by several bright lines. The observed magnitude then strongly depends on the spectral window used and on the sensitivity of the detector as a function of wavelength. In particular, it is important whether the radiation of $\mathrm{H}$ and $\mathrm{K}$ lines and of the infrared oxygen lines is included in the magnitude determination.

Knowing the meteor spectrum, the $\mathrm{V}$ magnitude in the international UBV photometric system can be computed from the formula (Allen, 1973):

$$
V=-2.5 \log \left(\int_{0}^{\infty} V_{\lambda} F_{\lambda} \mathrm{d} \lambda\right)-13.74
$$

The spectral flux $F_{\lambda}$ in $\operatorname{erg~cm}{ }^{-2} \mathrm{~s}^{-1} \AA^{-1}$ is related to the intensity $I_{\lambda}$ given in Fig. 3 by $F_{\lambda}-I_{\lambda} / R^{2}$, where $R-100$ $\mathrm{km} . V_{\lambda}$ is the $\mathrm{V}$ filter passband; it is greater than 0.1 between 4900 and $6400 \AA$. The emissions in blue and violet, including the $\mathrm{H}$ and $\mathrm{K}$ lines, do not influence the $\mathrm{V}$ magnitude. In fact no strong lines of the second component contribute to the $\mathrm{V}$ magnitude.

The $\mathrm{V}$ magnitude was used to compute $\mathrm{V}$-band luminous efficiency $\tau_{\mathrm{v}}$. The resulting values are given in Table 4 together with the V magnitudes. The average luminous efficiency is close to $\log \tau_{\mathrm{v}}=-11.9$ for both meteors. However, a systematic trend is present, the efficiencies being larger in the middle of the meteor bright part. At 
Table 4. V-Band luminous efficiencies of two Perseid meteors

\begin{tabular}{crrrrr}
\hline & \multicolumn{2}{c}{ Spectrum A } & & \multicolumn{2}{c}{ Spectrum B } \\
\cline { 2 - 3 } \cline { 5 - 6 } Point No. & \multicolumn{1}{c}{$V$} & $\log \tau_{\mathrm{v}}$ & & $V$ & $\log \tau_{\mathrm{v}}$ \\
\hline 1 & -10.10 & -12.0 & & -9.40 & -12.1 \\
2 & -10.71 & -12.0 & & -10.12 & -11.9 \\
3 & -9.54 & -11.8 & & -10.19 & -11.9 \\
4 & -10.27 & -11.7 & & -9.56 & -11.8 \\
5 & -9.88 & -11.9 & & -9.30 & -11.9 \\
6 & -10.54 & 11.9 & & & \\
7 & -9.85 & -11.9 & & & \\
8 & -9.32 & -12.0 & & & -11.93 \\
Average & & -11.89 & & \\
\hline
\end{tabular}

first sight it seems that this could be correlated with the strength of the second component. A more detailed evaluation, however, showed that the role of the second component in the variation of $\tau_{\mathrm{v}}$ is negligible. The situation is more complicated. Let us, for example, consider the fact that for a given mass of the radiating gas, higher temperature leads to higher intensity of spectral lines and hence to higher luminous efficiency. However, higher temperature also leads to higher ionization which causes a decrease of luminosity because the ions of such elements as $\mathrm{Fe}, \mathrm{Mg}, \mathrm{Na}$ have much fainter lines in the visual region than neutral atoms of the same elements. So, various effects acting in different directions are present even for the main component.

Our resulting average luminous efficiency is in surprisingly good agreement with the value of Ceplecha and McCrosky (1976). However, their value refers to meteor magnitude defined in a different way. In fact, V magnitude cannot be determined from normal non-spectral photographic meteor observation. Instead, the panchromatic magnitude, $M_{\mathrm{p}}$, is used to express meteor brightness and to compute photometric mass. Ceplecha (1987) gave the following formula for panchromatic magnitude of stars of normal spectral types :

$$
M_{\mathrm{p}}=V+0.62(B-V)-0.52(V-R) .
$$

Mctcor panchromatic magnitude is determined by measuring and comparing the images of meteor and stars. The emissions in the $3500-6600 \AA$ range contribute to meteor panchromatic magnitude.

We did not use spectral photographs to determine panchromatic magnitude because they are influenced by the presence of spectral grating. Instead, we took the results of classical photometry of meteor DMS-89046 (spectrum B) performed by van Oudheusden and van Dijk (1991). They used short focal length cameras with rotating shutters and gave the magnitude for individual shutter breaks separated by $0.04 \mathrm{~s}$, corresponding to about $2 \mathrm{~km}$ in height. This is a much lower resolution than we reached (Fig. 4), but we can compare their maximal magnitude at the height of $79 \mathrm{~km}\left(M_{\mathrm{p}}=-11.5\right)$ with our valuc in this region (points 2 and $3, V=-10.1$ ). This gives the "color index" $M_{\mathrm{p}}-V=-1.4$ and, correspondingly, the panchromatic luminous efficiency $\log \tau_{\mathrm{p}}=\log \tau_{\mathrm{v}}+0.56 \approx$ -11.4. The higher panchromatic efficiency is clearly caused by the presence of the $\mathrm{Ca}$ II lines and other bright emissions in blue and violet.
In summary, we recommend to use the panchromatic luminous efficiency of $\log \tau_{\mathrm{p}}=-11.4$ for Perseids (and other meteors with similar velocity) of brightness about -10 mag. Faint Perseids have probably different luminous efficiency owing to the lower relative brightness of the meteoritic lines in the second spectral components and larger role of the $\mathrm{N}_{2}$ bands. In any case, the luminous efficiency of fast meteors strongly depends on the photometric band used.

Our panchromatic value is about three times higher than that used previously (Ceplecha and McCrosky, 1976). Ceplecha (1996), analyzing the Lost City meteorite fall, found the luminous efficiency for velocity of $13 \mathrm{~km} \mathrm{~s}^{-1}$, ten times higher than previously used $\left(\log \tau_{\mathrm{p}}=-11.4\right.$ versus -12.4$)$. This was explained by the dependence of luminous efficiency on meteoroid mass, the higher value being valid for bodies in the $10 \mathrm{~kg}$ range, the lower for $10 \mathrm{~g}$ bodies. Our results indicate that the scale is to be adjusted also at high velocities, although the mass of the meteoroids studicd here is not particularly large. Using the above derived gas masses $m_{\mathrm{G}}$ and relaxation time $t_{\mathrm{R}}$, the initial mass of the meteoroid DMS-85016 was estimated to $80 \mathrm{~g}$ and of DMS-89046 to $40 \mathrm{~g}$.

Using the known spectrum, we can also compute the total radiative output of the meteor in all directions between two wavelengths, $\lambda_{1}$ and $\lambda_{2}$

$$
L_{\lambda_{1}, \lambda_{2}}=4 \pi \int_{\lambda_{1}}^{\lambda_{2}} I_{\lambda} \mathrm{d} \lambda
$$

$L$ is obtained in $\operatorname{erg~s}^{-1}$ and can be directly compared with the kinetic energy loss rate computed as above. For example, at point 6 in spectrum $\mathrm{A}$, the loss of kinetic energy is $3 \times 10^{16} \mathrm{erg} \mathrm{s}^{-1}$. It was found that $2.4 \%$ of this value is radiated out between 3500 and $6600 \AA$. A total of $1.4 \%$ is due to the $\mathrm{CaII} \mathrm{H}$ and $\mathrm{K}$ lines, $0.6 \%$ by the radiation above $4000 \AA$ and $0.4 \%$ by the radiation below $3900 \AA$. These data are not particularly certain owing to the uncertain calibration of $\mathrm{Ca}$ II lines, but the large role of the radiation in the violet part of the spectrum is obvious.

\section{Conclusions}

We have observed and analyzed two spectra of bright Perseids. Three main aspects have been studied-the relation of two spectral components, chemical composition of the meteoroids, and meteor luminous efficiency. In addition, a qualitative explanation of the Russcl (1960) cffect in metcor flares was presented.

Two spectral components were identified in agreement with previous findings (Borovička, 1994). The temperature of the main component changed in the range $4400-4800 \mathrm{~K}$, the second component is consistent with the temperature of $10,000 \mathrm{~K}$. 'T he mass of the radiating gas fluctuated, causing the fluctuation of the meteor brightness. The ratio of the second component to the main component gas varied as well, though more smoothly: from about $1: 100$ at the beginning to $1: 15$ in the middle and $1: 30$ at the end of the bright part of the meteors. This ratio proved to be not an unambiguous function of meteor velocity, height and brightness; it depends on the previous evolution of ablation. The most probable interpretation 
is that the enhancement of the second component is a consequence of the development of a strong ablation shock wave. On the other hand, infrared meteor observation show that atmospheric lines behave differently and that $\mathrm{a} \approx 10,000 \mathrm{~K}$ air is present also at the beginning. More theoretical studies are needed to clarify these observational evidences.

In contrast to slower meteors, ablated calcium was completely evaporated in the observed Perseids. Within the observational error, i.e. within a factor of two, the elemental ratios of $\mathrm{Fe}, \mathrm{Na}, \mathrm{Mg}, \mathrm{Si}, \mathrm{Ca}, \mathrm{Cr}$, and $\mathrm{Mn}$ were found consistent with the abundances in carbonaceous chondrites and comet Halley. This is not surprising for cometary meteoroids. The same conclusion was made for $\mathrm{Fe}$, $\mathrm{Na}, \mathrm{Mg}$, and $\mathrm{Ca}$ in Giacobinid meteors by Millman (1972) on the basis of a quite different method. More interestingly, the hydrogen content proved significantly lower than in comet Halley dust and probably lower than in carbonaceous chondrites. This can be explained by a loss of volatiles when the meteoroids approached the Sun to $0.9 \mathrm{AU}$ at perihelion.

The V-band luminous efficiency was found to vary in the range $\log \tau_{\mathrm{v}}=-11.8$ to -12.0 in magnitude based/c.g.s. units. The panchromatic luminous efficiency is higher owing to the presence of bright lines of $\mathrm{Ca} \mathrm{II} \mathrm{in}$ the violet. We recommend to use the value of -11.4 for very bright Perseids. This value is three times higher than that used up to now. It was estimated that about $1.5 \%$ of meteoroid kinetic energy was radiated out in the Ca II lines and $1 \%$ in all other lines between 3500 and $6600 \AA$.

Acknowledgements. We acknowledge the use of the electronic form of the table from Sviderskiene (1988) at CDS, Strasbourg. The first author was supported by grant No. 303406 from the Academy of Sciences of the Czech Republic and by grant No. 1862 from the Grant Agency of the Czech Republic.

\section{References}

Allen, C. W. (1973) Astrophysical Quantities. Althone Press, London.

Anders, E. and Grevesse, N. (1989) Abundances of the elements : meteoritic and solar. Geochim. Cosmochim. Acta 53, 197214.

Betlem, H. and de Lignie, M. (1985) Twee vuurboltrajekten. Radiant, J. Dutch Met. Soc. 7, 126-128.

Borovička, J. (1993) A fireball spectrum analysis. Astron. Astrophys. 279, 627-645.
Borovička, J. (1994) Two components in meteor spectra. Planet. Space Sci. 42, 145-150.

Borovička, J. and Boček, J. (1995) Television spectra of meteors. Earth, Moon, and Planets 71, 237-244.

Borovička, J. and Spurný, P. (1996) Radiation study of two very bright terrestrial bolides and an application to the comet S-L 9 collision with Jupiter. Icarus 121, 484-510.

Ceplecha, Z. (1964) Study of a bright meteor flare by means of emission curve of growth. Bull. Astron. Inst. Czechosl. 15, 102-112.

Ceplecha, Z. (1987) Geometric, dynamic, orbital and photometric data on meteoroids from photographic fireball networks. Bull. Astron. Inst. Czechosl. 38, 222-234.

Ceplecha, Z. (1996) Luminous efficiency based on photographic observations of Lost City fireball and implications to the influx of interplanetary bodies onto Earth. Astron. Astrophys. 311, 329-332.

Ceplecha, Z. and McCrosky, R. E. (1976) Fireball end heights: a diagnostic for the structure of meteoritic material. $J$. Geophys. Res. 81, 6257-6275.

Cook, A. F., Halliday, I. and Millman, P. M. (1971) Photometric analysis of spectrograms of two Perseid meteors. Can. J. Phys. 49, 1738-1749.

Halliday, I. (1961) A study of spectral line identifications in Perseid meteor spectra. Publ. Dominion Obs. Ottawa 25(1), 3-16.

Halliday, I. (1969) A study of ultraviolet meteor spectra. Publ. Dominion Obs. Ottawa 25(12), 315-322.

Halliday, I. (1987) The spectra of meteors from Halley's comet. Astron. Astrophys. 187, 921-924.

Harvey, G. A. (1973a) Spectral analysis of four meteors. In Evolutionary and Physical Properties of Meteoroids, eds C. L. Hemenway, P. M. Milman and A. F. Cook, NASA-SP 319, pp. 103-129.

Harvey, G. A. (1973b) Elemental abundance determinations for meteors by spectroscopy. J. Geophys. Res. 78, 3913-3926.

Jessberger, E. K., Christoforidis, A. and Kissel, J. (1988) Aspects of the major element composition of Halley's dust. Nature 322, 691-695.

Kokchirova, G. I. (1993) The quantitative analysis of the Perseid meteor spectra. Asiron. Vestnik 27(3), 100-112.

Millman, P. M. (1972) Giacobinid meteor spectra. J. R. Astron. Soc. Canada 66, 201-211.

Millman, P. M. and Halliday, I. (1961) The near-infra-red spectrum of meteors. Planet. Space Sci. 5, 137-140.

Millman, P. M., Cook, A. F. and Hemenway, C. L. (1971) Spectroscopy of Perseid meteors with an image orthicon. Can. J. Phys. 49, 1365-1373.

Russel, J. A. (1960) A time resolved spectrum of the terminal burst of a Perseid meteor. Astrophys. J. 131, 34-37.

Sviderskiene, Z. (1988) Mean energy distributions in the stellar spectra of different spectral types and luminosities. I. Normal stars. Bull. Vilnius Obs. 80, 7-104.

van Oudheusden, J. P. and van Dijk, M. (1991) Astrometrisch en fotometrisch onderzoek aan de vuurbol van 13 augustus $1989,2^{\mathrm{h}} 27^{\mathrm{m}} 40^{\mathrm{s}}$ UT. Radiant, J. Dutch Met. Soc. 13, 36-41. 